\title{
LA CONSTRUCCIÓN CULTURAL DE LAS RELACIONES LABORALES. UNA PERSPECTIVA DESDE LA ANTROPOLOGÍA DEL TRABAJO
}

\section{BUILDING CULTURAL AND LABOUR RELATIONSHIPS: A PERSPECTIVE FROM ANTHROPOLOGY OF WORK}

\author{
Miguel GonZÁLEZ GonZÁLEZ \\ Universidad de León \\ migog@unileon.es
}

Recibido: 06/06/2017

Aceptado: 13/09/2017

RESUMEN: Este artículo analiza el ámbito laboral de la sociedad actual desde la perspectiva de la antropología del trabajo. El criterio es construir herramientas de análisis que metodológicamente sean útiles para abordar el fenómeno laboral desde una perspectiva aplicada. Los resultados a partir del estudio de cuestiones como las relaciones sociales desde la obtención de alimentos, los distintos niveles laborales de las empresas productivas, la división del trabajo, la explotación laboral y la organización del trabajo, la antropología industrial o la cultura de empresa, muestran un amplio campo que la antropología del trabajo tiene por explorar para analizar y explicar las relaciones laborales de hoy en día.

PALABRAS CLAVE: Trabajo; Antropología del trabajo; Cultura del trabajo; Explotación laboral.

ABSTRACT: This article analyzes the work environment of the current society from the perspective of the anthropology of work. The criterion is to build analysis tools that are useful methodologically to address the labor phenomenon from an applied perspective. The results from the study of issues such as social relations, the different levels of work of productive organizations, division of labor, labor exploitation, work organization, industrial anthropology or organizational culture show a broad field that the anthropology of work has to explore, analyze and explain current occupational relationship.

KEYWORDS: Labor; Anthropology of work; Culture of work; Labor exploitation.

\section{INTRODUCCIÓN.}

El estudio del ámbito laboral en sociedades industriales ha estado copado generalmente por disciplinas como el Derecho, la Sociología o la Economía, mientras 
la Antropología se ocupaba de los patrones y procesos laborales en sociedades tradicionales y preindustriales, rurales y campesinas. Sin embargo, a partir de los años 70 del pasado siglo XX, la antropología entró en el mundo industrial y de la empresa, poniéndose énfasis en las "culturas del trabajo". En este contexto, el objetivo general de la investigación aquí mostrada es analizar el proceso laboral en la sociedad actual desde una perspectiva de la Antropológica del trabajo. Además, pretendemos comprender y explicar la experiencia laboral como proceso productivo cotidiano, así como construir herramientas de análisis que metodológicamente sean útiles para abordar el fenómeno laboral en la sociedad actual desde una perspectiva aplicada.

En este argumento en primer lugar planteamos cómo desde las actividades para la satisfacción de necesidades básicas como puede ser la obtención de alimentos existen unas relaciones de producción que contribuyen al proceso productivo, a la vez que actúan como marcador de identidad, en tanto ocupa un lugar primordial en la vida de las personas. Después abordamos la división del trabajo, ya sea por edad o género, lo que otorga un valor diferencial al trabajo, así como factores que determinan las diferencias de género en el trabajo, lo que originará una segregación vertical que constituye la construcción de las desigualdades entre hombres y mujeres. A continuación, un breve estudio en la historia reciente de la relación entre trabajo y sistemas de producción a través de los teóricos de la sociedad industrial capitalista, nos muestran un trabajador visto como mano de obra más que como persona, o a través de los pragmáticos de la razón industrial, trabajadores convertidos en recursos humanos para dar un orden tecnocrático al sistema productivo. Abordamos así mismo, la repercusión en cuestiones como el trabajo de los niños o explotación infantil, o el debate sobre prestación de servicios y contraprestación, en sus diversas formas, lo que nos permite entrar en cuestiones como el salario, protección y derecho laboral, que nos sitúan en los momentos más recientes, y de nuevo, en la construcción de la identidad. Por último, se analizan las organizaciones y la cultura organizacional como una trama simbólica de significados compartidos que vertebra al grupo. En este apartado, núcleo central de debate o discusión de la aportación realizada en esta investigación, partimos de nociones clásicas sobre el concepto de cultura para llegar a la definición de cultura organizacional; retomamos nociones clásicas como el "taylorismo", "la organización formal" y la "burocracia racional" para abordar el actual liderazgo, la antropología industrial y de la empresa, y las modernas "culturas del trabajo". Así mismo, planteamos algunas cuestiones para la reflexión y aspectos para seguir avanzando en la investigación de la situación laboral en la sociedad actual. 


\section{DE LA RECOLECCIÓN A LA PRODUCCIÓN.}

Riezink $^{1}$ define el trabajo como la posibilidad que tiene el hombre de adecuar especialmente el entorno a sus necesidades como condición de su misma supervivencia. La necesidad de obtención de alimentos resulta prioritaria frente a otras actividades importantes para la supervivencia. Ninguna otra actividad como la reproducción, el control social o la transmisión de conocimientos sería posible sin la energía derivada de los alimentos. Las actividades de obtención de esos alimentos son de tal transcendencia, que la forma en que una sociedad obtiene su alimento tiene un fuerte valor predictivo en otros aspectos de su cultura. En la antigüedad, aunque el número de sociedades que subsistían de la caza y la recolección era grande, el número de personas de cada sociedad era pequeño, puesto que ningún entorno podría soportar una gran población únicamente con sus plantas y animales salvajes. En este tipo de sociedades cazadoras-recolectoras la economía de la sociedad y del grupo doméstico apenas se diferenciaba, y en este sentido, desarrollaron esquemas de reparto y redistribución de los recursos que poseían entre otros miembros de la comunidad. Con la domesticación de los animales los hombres descubrieron el pastoreo como una extensión de la caza así como la agricultura fue una extensión de la recolección. Engels ${ }^{2}$ en la obra "El papel del trabajo en la transformación del mono en hombre" (1876) afirma que el trabajo es la condición básica y fundamental de toda vida humana, que el trabajo ha creado al propio hombre. El origen y la difusión de los sistemas de obtención de alimentos condujeron a la formación de sistemas políticos y sociales cada vez más complejos, de forma que la producción de alimentos condujo a grandes cambios en la vida humana. En este sentido hay que precisar que las materias no son recursos si las personas no pueden utilizarlas, y es precisamente la cultura la que identifica los elementos de nuestro entorno que son recursos y los que no. Según Eric Wolf ${ }^{3}$, la noción de trabajo social nos hace posible conceptualizar los caminos más importantes en los que los seres humanos organizan su producción. Una serie de relaciones sociales ocurren históricamente, en las que el trabajo se desarrolla para arrancar energía a la naturaleza por medio de herramientas, destreza, organización y conocimientos. Como apunta Narotzky ${ }^{4}$, las relaciones sociales de producción son el resultado del modo en que la cooperación, bien voluntaria o bien forzada, y la coordinación se llevan a cabo entre quienes contribuyen, de un modo u otro al proceso productivo. De esta manera, el

\footnotetext{
1 RIEZNIK, P. (2001). “Trabajo, una definición antropológica”. Razón y revolución, 7, p.1

2 ENGELS, F. (2011). El papel del trabajo en la transformación del mono en hombre. Valencia: NoBooks Editorial.

3 WOLF, E. R. (1987). Europa y la gente sin historia. Fondo de Cultura Económica.p.75.

4 NAROTZKY, S. (2004). Antropología económica. Nuevas tendencias. Santa Cruz de Tenerife: Melusina. p.57.
} 
análisis del proceso laboral puede ayudarnos a comprender la experiencia cotidiana del trabajo. Palenzuela y Lozano ${ }^{5}$ definen el trabajo como toda acción que tiene el objetivo económico principal de producir bienes o prestar servicios y señalan además, que el trabajo es marcador de la identidad, está ligado a la construcción de comportamientos e ideas y ocupa un lugar principal en la vida de las personas. Por lo tanto podríamos argumentar que en esa actividad productora, fundamental para la subsistencia del ser humano, el trabajo es un factor importante en la forja de su identidad. En este aspecto, adelantamos, para nuestra argumentación posterior que, como apunta Munárriz ${ }^{6}$, el individuo no se comporta en la vida cotidiana ejerciendo una sola identidad, sino que éstas se entrecruzan; a la identidad personal que nos singulariza a través de nuestros rasgos físicos, biológicos y psíquicos se une la identidad social basada en el conjunto de creencias, sentimientos o costumbres que compartimos con los miembros del grupo social al que pertenecemos.

\section{LA DIVISIÓN DEL TRABAJO.}

Bohannan ${ }^{7}$ señala que el trabajo es un mecanismo para convertir los recursos (incluyendo los humanos) en formas $u$ organizaciones que aumenten las posibilidades de supervivencia y el placer de vivir. En todas las sociedades existe por distintos motivos una división sexual del trabajo que mayoritariamente es por sexo y edad, pero en sociedades con una especialización ocupacional permanente, además de esta división existen otras en función de la clase social, la riqueza o el poder, dando lugar a la estratificación social. En esta línea, Durkheim ${ }^{8}$ apuntaba que la división del trabajo no es exclusivo del mundo económico; se puede observar su influencia creciente en las regiones más diferentes de la sociedad, se especializan las funciones políticas, administrativas y judiciales así como las funciones artísticas y las científicas. Ya Adam Smith" en "La riqueza de las naciones" (1776) propugnó la división del trabajo como el elemento primordial del aumento de la productividad y la riqueza.

LOZANO, M.J. \& PALENZUELA. P. (2016). “Trabajo y culturas del trabajo en la globalidad hegemónica". Revista Andaluza de Antropología, 11, pp.1-15.

6 MUNÁRRIZ, L. Á. (2009). “La identidad personal en la Región de Murcia”. Revista murciana de antropología, 16, pp. 309-324.

BOHANNAN, P. (1996). Para raros, nosotros. Madrid: Ediciones AKAL. p.112.

DURKHEIM, E. (1987). La división del trabajo social (Vol. 39). Madrid: Ediciones Akal.

9 SMITH, A. (1994). Investigación sobre la naturaleza y causas de la riqueza de las naciones. México: Fondo de Cultura Económica. 
El trabajo que debe ser realizado en cualquier sociedad, está siempre dividido entre sus miembros y en sociedades simples, como las mencionadas en el apartado anterior, de cazadores-recolectores, la división primaria del trabajo se efectúa entre hombre y mujer y a medida que una cultura se hacía más compleja y se utilizaban nuevos recursos, la división del trabajo se trasladaba a otras esferas. Basta recordar una obra pionera en la moderna antropología social como es "Los Argonautas del Pacífico Occidental" (1922) de Malinowski ${ }^{10}$ dedicado precisamente a las relaciones económicas y a las formas de competencia y de intercambio de los habitantes de las islas Trobriand donde describe perfectamente la división sexual del trabajo. En su vida tribal, el status de las mujeres es muy elevado. En general no participan en los consejos de los hombres, pero tienen sus propias reuniones para muchos asuntos y controlan determinados aspectos de la vida tribal. Así, por ejemplo, parte del trabajo hortícola está bajo su control, y esto más bien se considera un privilegio que un deber; también se cuidan de ciertas secuencias de las grandes reparticiones ceremoniales de alimentos, relacionadas con el ritual funerario de los boyowas, etc. ${ }^{11}$

Por otra parte, como plantea Rosaldo ${ }^{12}$, es evidente que los hombres y las mujeres son biológicamente diferentes y que estas diferencias se manifiestan no solamente en sus órganos genitales y reproductores sino que también en sus características sexuales secundarias como la voz o el pelo corporal. Las diferencias de sexo son biológicas, pero el género, como dice esta autora, abarca todos los rasgos que una cultura atribuye e inculca a hombres y mujeres. Es decir, el género se refiere a la construcción cultural de las características masculinas y femeninas. A partir de esa construcción cultural se asignan las tareas o actividades que cada cultura considera adecuados para desempeñar cada persona en función de su género y nacen por lo tanto los roles de género y con ellos los estereotipos. Esta asignación de roles ha llevado a las mujeres a lo largo de la Historia a un plano de desigualdad que sigue vigente hoy en día. A partir de esa estratificación de género, el trabajo que desarrollan hombres y mujeres ha sido tradicionalmente diferente, produciéndose en el caso de las mujeres el desempeño de tareas peor remuneradas y con menor prestigio social. Según Bourdieu ${ }^{13}$, la división sexual es un principio básico de la violencia simbólica de la estructura social. Lo que Bourdieu denomina la dominación masculina presupone actividades y actitudes de las

\footnotetext{
${ }^{10}$ MALINOWSKI, B. (1986). Los Argonautas del Pacifico Occidental. Barcelona: Península, p.69.

11 GODELIER, M. (1976). Antropología y economía. Barcelona: Anagrama.

12 ROSALDO, M. Z. (1980). "The use and abuse of anthropology: reflections on feminism and cross-cultural understanding". Signs, 5(3), pp. 389-417.

13 BOURDIEU, P. (1998). La dominatión masculine. Paris: Le Seuil.
} 
mujeres y los hombres marcadas por su género que a través de lo simbólico perpetúan ese principio de diferenciación.

En las relaciones de trabajo de los siglos XIX y XX hubo una interacción entre el patriarcado y el capitalismo, que otorgó un valor diferencial al trabajo en función del género. El género por lo tanto divide espacios laborales diferenciados, dando como resultado trabajos masculinizados y feminizados.

Reskin y Padavic ${ }^{14}$ señalan tres factores que determinan las diferencias de género en el trabajo que señalan: primero, la división sexual en el trabajo, considerada como la asignación de tareas basada en el sexo de los trabajadores; segundo, el mayor valor otorgado al trabajo realizado por los hombres en relación al que realizan las mujeres y tercero, la construcción del género en el trabajo por parte de empresarios y trabajadores. Esta diferenciación en las tareas asignadas en función del género y que suponen una distinta valoración social, económica y simbólica se manifiesta según Abasolo y Montero ${ }^{15}$ en una desigualdad entre mujeres y hombres, y esta división sexual en el trabajo es lo que origina la segregación horizontal.

Por otra parte, desde la perspectiva del feminismo y de la antropología feminista se prefiere el término relaciones de producción al de modo de producción, debido principalmente a su similitud con el enfoque "relaciones de género". Desde esta perspectiva, Henrietta Moore ${ }^{16}$ señala que los antropólogos deben fijarse en lo que la gente, las mujeres en particular, hacen, tanto como en lo que dicen que hacen, teniendo en cuenta que las mujeres no son iguales en todas partes, lo que significa ser mujer u hombre depende de la cultura considerada, puesto que no es lo mismo hablar de "mujeres" que añadir roles como "musulmana, asiática, de clase media, etc.". La complejidad de este tipo de roles, por lo tanto, es lo que construye su "personalidad social".

En la asignación de tareas según el binomio sexo-género nos encontramos con el valor diferencial de las mismas. Las tareas que reciben más valoración, prestigio y salario son las asignadas a los varones y al contrario las asignadas a las mujeres. Por otra parte, las identidades de género establecen relaciones de poder y de jerarquización social, que producen una jerarquización vertical procedente del sistema patriarcal. Según Raquel Osborne ${ }^{17}$ una de las dicotomías más extendidas en casi todas las

${ }^{14}$ RESKIN, B. F., \& PADAVIC, I. (1994). Men and women at work. Thousand Oaks: Pine ForgePress.

15 ABASOLO, O. \& MONTERO, J. (2012). "Trabajos: empleo, cuidado y división sexual del trabajo". Guía de ciudadanía con perspectiva de género. Madrid: Fuhem Ecosocial.

${ }^{16}$ MOORE, H. L. (2009). Antropología y feminismo. Madrid: Cátedra.

${ }^{17}$ OSBORNE, R. (1993). La construcción sexual de la realidad: un debate en la sociología contemporánea de la mujer. Valencia: Universitat de Valencia. 
sociedades es la de naturaleza-cultura, asociándolo con mujer-hombre, lo que explica la devaluación de la mujer, y la prueba de ello es la disparidad de funciones asignadas a cada sexo según la historia y la antropología, lo que ha provocado la división del trabajo en los términos asimilados actualmente. En este sentido y como señala también Comas ${ }^{18}$ la división sexual del trabajo es un rasgo universal aunque varíe la forma que adopta entre unas sociedades y otras. La división del trabajo jerarquiza las tareas pero también provoca la jerarquización de las personas, por lo que el trabajo integra las distintas formas de desigualdad social y por ello es la clave para entender los sistemas de género y su articulación con otras divisiones sociales. Para Comas la construcción social del género y su vinculación con la división del trabajo se refleja en una constelación de ideas y de símbolos que denomina "modelos de representación" sobre género y trabajo que se concretan de forma distinta en cada sociedad, ya que han de ser compatibles con los modelos institucionales existentes y en concreto con las relaciones productivas. Estos modelos de representación están constituidos por las nociones y símbolos acerca de las diferencias entre hombres y mujeres y de lo que es trabajo o no lo es. En este sentido la casa, la vida familiar se percibe como el mundo de las mujeres, y la calle y las relaciones sociales se entienden de dominio masculino. La vinculación de las mujeres a la vida doméstica implica que se les atribuye la realización de todas las tareas relacionadas con el crecimiento humano. No hay que olvidar que la familia es una institución clave para enmarcar la división sexual del trabajo y asegurar la reproducción social de los trabajadores, lo que la convierte en el marco para explicar los roles atribuidos a las mujeres, con lo que nos encontramos que al trabajo productivo realizado por las mujeres se une el reproductivo, doméstico, el realizado en el ámbito privado y que genera beneficios que en la mayoría de las ocasiones no se han tenido en cuenta.

\section{LA EXPLOTACIÓN LABORAL Y LA ALIENACIÓN.}

La relación entre trabajo y sistemas de producción se puede comprobar a lo largo de la historia observando las diferentes condiciones en las que el hombre ha estado sujeto al trabajo: la esclavitud, la servidumbre, con el vasallo sujeto a la tierra y al señor feudal. Posteriormente con la revolución industrial el hombre se transformará en obrero, proletario para distinguirse del dueño de los medios de producción.

${ }^{18}$ COMAS D’ARGEMIR, D. (1995). Trabajo, género, cultura: la construcción de desigualdades entre hombres y mujeres. Barcelona: Icaria. p.17. 
Desde el primer tercio del siglo XIX, los teóricos de la sociedad industrial apuntaron su análisis hacia las fábricas y sus obreros. Autores como Marx, Engels, Durkheim o Weber, entre otros, estudiaron desde distintas ópticas lo que implicaba la materialización de la nueva utopía industrial. Como apunta Cuesta ${ }^{19}$ si por un lado las nuevas sociedades industriales capitalistas demostraban una eficiente capacidad de generación de riquezas, también introdujeron enormes desigualdades económicas, sociales y políticas que acrecentaron el conflicto entre burguesía y proletariado. La propia fábrica se convirtió en un espacio máximamente coercitivo y mínimamente participativo, donde la férrea administración del patrón y la represión practicada por sus capataces fueron la norma general y la tónica en la relación entre clases dominadoras y dominadas.

En el siglo XIX la concepción marxista plantea la distinción entre el concepto de trabajo y el de "trabajo alienado". Bajo relaciones sociales asimétricas y de dominación el trabajo pierde su valor fundamental de constituir la articulación entre la actividad material y la intelectual, valor que solo se puede recuperar en una sociedad sin clases. Para Isidoro Moreno ${ }^{20}$ fue muy importante el papel tanto del marxismo como de otras corrientes del socialismo utópico en la dignificación del trabajo a ojos de quienes lo sufrían, sin que ello supusiera legitimar las formas y condiciones en que éste se desarrollaba. En sus Manuscritos económico-filosóficos de 1844, Marx ${ }^{21}$ señala que la alienación del hombre y, en general, todas las relaciones en las que el hombre se encuentra consigo mismo, solo se realizan y se expresan a través de la relación que el hombre se encuentra con otro hombre. Así pues, a través del trabajo alienado, enajenado, el trabajador genera la relación con el trabajo de un hombre que es ajeno a dicho trabajo, que se encuentra fuera de él. Considera que desde el punto de vista capitalista el trabajador no es una persona en sí misma, sino una mano de obra que puede representarse en su equivalente económico, es decir, el trabajador es dinero utilizable como mano de obra para multiplicarse.

Con los "pragmáticos de la razón industrial" (Tylor, Fayol, Stajanov, Ford...) se pusieron en práctica los conocimientos de la ingeniería sobre unos sujetos-objetos deshumanizados convertidos en recursos humanos para dar un orden tecnocrático al sistema productivo. A través de la llamada organización científica del trabajo se introdujo una mayor racionalización y optimización de la empresa industrial a costa

${ }^{19}$ CUESTA ÁVILA, R. (2016). "Debates teóricos sobre el 'fin de la sociedad industrial' en el horizonte laboral del nuevo milenio". Revista Andaluza de Antropología, 11, pp. 222-248.

${ }^{20}$ MORENO, I. (1997). “Trabajo. Ideologías sobre el trabajo y culturas del trabajo". Revista Andaluza de Relaciones Laborales, 3, pp. 9-28.

${ }^{21}$ MARX, K. (2004). Manuscritos económico-filosóficos de 1844. Buenos Aires: Ediciones Colihue, pp.116-117. 
de una incapacidad para generar y gestionar dinámicas democráticas, que resultaban incompatibles con un sistema absolutamente productivista ${ }^{22}$. A través de la misma, se profundiza en la división del trabajo y mediante la división o el fraccionamiento de las tareas productivas y el estudio de los movimientos y tiempos, en función del cual se definen los tiempos de trabajo, se definen los puestos de trabajo, y se evalúa la productividad. En los "Principios de la administración científica" de 1891, Tylor ${ }^{23}$ entiende que la naturaleza humana es tal, que muchos obreros si fueran abandonados a sí mismos, prestarían poca atención a las instrucciones escritas, y piensa que la tendencia del obrero es trabajar con ritmo lento y que solo con la conciencia o la presión externa adopta un ritmo más rápido.

En el contexto actual en el que nos encontramos en el mundo en pleno siglo XXI son frecuentes las formas de esclavitud moderna que afectan a millones de personas en el mundo. A pesar de que casi todos los países la consideran ilegal, la esclavitud disfrazada en alguna de sus formas sigue existiendo, sobre todo en los países llamados del tercer mundo, donde las personas se ven forzadas a trabajar jornadas de hasta 20 horas sin recibir ningún pago, víctimas de esclavitud sexual, personas obligadas a la mendicidad forzada o el trabajo de menores. Además de ello son generales los casos de explotación laboral también en países desarrollados, que normalmente recaen en los grupos más débiles como son las mujeres, o los inmigrantes sin papeles.

En los procesos de deslocalización industrial de la segunda mitad del siglo $\mathrm{XX}$ en los países occidentales y la re-localización en terceros países emergentes principalmente del Sudeste asiático, China, América Latina, África y el Este Europeo se han aplicado las mismas técnicas del taylorismo y el fordismo ya que resultaban anacrónicas en los países occidentales, provocando importantes consecuencias sociales, económicas y políticas en las vidas de todos los habitantes del planeta. Así mismo, esta deslocalización industrial ha producido una terciarización en los países occidentales, con una tendencia a los "servicios industrializados" dentro de un mercado laboral que como indica Cuesta Avila ${ }^{24}$ encontramos una dualización entre el "posttrabajo" de alta cualificación, propio de los empleados del conocimiento y altamente creativos, frente al "hiper-trabajo" de baja cualificación, asociado a los empleados de la información, altamente reproductivos y que siguen sojuzgados a los mismos métodos

\footnotetext{
${ }^{22}$ Buen ejemplo de la organización científica del trabajo lo tenemos en el cine de principios del siglo XX como por ejemplo en la película "Tiempos modernos" (1936) escrita y dirigida por Charles Chaplin, donde se pueden observar escenas que ilustran las características de los modelos taylorista y fordista. CHAPLIN, C., CHAPLIN, C. (1936). Modern Times. Estados Unidos: United Artists.

23 TAYLOR, F. W. (1969). Principios de la Administración Cientifica. México: Herrero Hnos. S. A.

${ }^{24}$ CUESTA ÁVILA, R. (2016). "Debates teóricos sobre el 'fin de la sociedad industrial' en el horizonte laboral del nuevo milenio". Revista Andaluza de Antropología, 11, pp. 215-216.
} 
de control de la fábrica convencional a través de métodos de coerción simbólica. En este sentido Parker y Slaughter ${ }^{25}$ estudiaron las prácticas productivas japonesas de "dirección por estrés" donde se mejoraba la productividad gracias a la reducción de tiempos y la disminución de personas asignadas a una determinada tarea, lo que desde la perspectiva de los trabajadores no es más que una forma de explotación, donde los propios trabajadores se convierten en cómplices de su propia explotación al identificar puntos débiles y efectuar recomendaciones para tomar acciones correctivas, lo que conlleva a presionar el ritmo. Para Ulrich ${ }^{26}$, cuando se exige más a los empleados, que sean más globales, más flexibles, más productivos, más dispuestos a trabajar en equipo, muchas veces son reclamos reales e inevitables, pero tienen efectos como pueden ser la depresión de los mismos, y sienten que se les pide que hagan más de lo que pueden con los recursos que cuentan. Por lo tanto habría que resolver los desequilibrios entre recursos y exigencias.

En el caso del trabajo y explotación infantil, que es una realidad en algunas sociedades actuales, debemos distinguir dos situaciones ${ }^{27}$ : por una parte encontramos casos de niños que tienen que trabajar de una forma más o menos estable para contribuir al mantenimiento de sus grupos familiares bien en la calle o en el interior de las casas de una forma más invisible complementando la actividad doméstica de sus padres o adultos con los que viven o en pequeños talleres y que en ocasiones resultan fundamentales para asegurar la reproducción de la unidad familiar, y por otra parte el trabajo que realizan los niños en empresas formales en las cuales cumplen una serie de exigencias de tipo laboral pero que en la mayor parte de los casos no están reconocidos con ningún tipo de contrato o acuerdo. En otros casos se pueden identificar otras formas de trabajos infantiles absolutamente alienantes y dañinos para los niños, especialmente relacionadas con la delincuencia y prostitución infantil.

\section{EL INTERCAMBIO: LA PRESTACIÓN DEL TRABAJO Y EL SALARIO.}

Desde un punto de vista etimológico la palabra "sueldo", según G. Gual ${ }^{28}$ procede del adjetivo solidus, que significa sólido o compacto, que se decía de las monedas de

\footnotetext{
${ }_{25}$ PARKER, M. y SLAUGHTER, J. (1988) Choosing Sides: Unions and the Team Concept, Detroit: Labor Notes.

${ }^{26}$ ULRICH, D. (1997). Recursos humanos champions. L'Hospitalet de Llobregat: Ediciones Granica SA. pp. 211214.

${ }^{27}$ MAREIRA ESTRADA, F. (2002). “Trabajo Infantil: algunas consideraciones desde la antropología”. Rev. austral cienc. soc, 6, pp. 113-124.

28 GARCÍA GUAL, C. (1990). "En busca de los orígenes: algunas etimologías de términos económicos”. Revista de economía, 5, p. 114.
} 
oro en el Bajo Imperio y que posteriormente se convirtió en latín vulgar en el soldus, que derivó al castellano en sueldo. Quienes recibían un sueldo en un primer momento eran los soldados y también a ellos se designaba la paga conocida como salarium, el "salario", una cantidad para la compra de sal. Hoy en día atendiendo al concepto legal y según señala el art. 26.1 del Estatuto de los trabajadores ${ }^{29}$, se considera salario a la totalidad de percepciones económicas de los trabajadores, sin discriminación por razón de sexo, en dinero o en especie, por la prestación profesional de sus servicios laborales por cuenta ajena, ya retribuyan el trabajo efectivo, cualquiera que sea la forma de remuneración, o los períodos computables como de trabajo.

Considerando que la economía es un sistema de producción, distribución y consumo de recursos, nos encontramos que tradicionalmente los economistas se han centrado en el estudio de los países modernos y en los sistemas capitalistas mientras que entre los antropólogos se ha creado el debate en antropología económica entre "formalistas" y "sustantivistas". Mientras los formalistas (Herskovits, Firth, Leclair, Schenider o Burling) se declaran partidarios de la aplicabilidad de la teoría económica a todas las sociedades, para los sustantivistas (Malinowski, Thurnwald, Polanyi, Dalton o Sahlins) las instituciones económicas tienen que ser estudiadas en cada caso: la teoría económica no es de aplicación general, sino particular y por tanto sustantiva, empleando tradicionalmente la recogida de datos en economías no industriales. Como señala Molina $^{30}$ en el fondo del debate se halla en realidad una discrepancia acerca de la visión de la ciencia: una deductiva y formal (formalismo) y otra empírica e inductiva (sustantivismo).

Como hemos apuntado, un modo de producción es una forma de organizar la producción, un conjunto de relaciones sociales a través de las cuales se despliega la fuerza de trabajo. Teniendo en cuenta que en el sistema capitalista de producción el dinero compra la fuerza de trabajo se produce frecuentemente una fractura entre los actores implicados en el proceso de producción, en este caso empleadores y empleados. Sin embargo, en las sociedades no industriales, el trabajo no se puede comprar sino que se da como una obligación social basada en el parentesco, la ayuda mutua para la producción.

Para antropólogos como Maurice Godelier o Claude Meillassoux el concepto de "modo de producción" se puede aplicar a cualquier forma social. Lo definieron como conjuntos de fuerzas productivas (factores de producción) y relaciones de producción.

${ }^{29}$ Real Decreto Legislativo 2/2015, de 23 de octubre, por el que se aprueba el texto refundido de la Ley del Estatuto de los Trabajadores.

${ }^{30}$ MOLINA, J. L. (2004). Manual de antropología económica. Bogotá: UAB. p.47. 
Godelier $^{31}(1967,1977)$ argumenta que se trata de un proceso doble, por una parte "el trabajo como relación entre hombres y naturaleza" y por otra, la "relación de los hombres entre sí". Producir es combinar tres categorías de realidad: los recursos u objetos sobre los que actúa el trabajo, un conjunto de medios y de instrumentos que actúan sobre esos recursos y el trabajo del hombre. Pero esta actividad económica no se puede separar de su marco social que es la unidad de producción, y que puede ser la explotación familiar o la empresa industrial. Este marco hace que aparezcan relaciones entre individuos y grupos, más o menos solidarias o más o menos conflictivas en lo que respecta al acceso a los medios de producción y su control. Y estas relaciones de producción están condicionadas por los propios medios de producción. Así, según Godelier" "al conseguir nuevos medios de producción, los hombres cambian su modo de producción y, al cambiar éste, cambian todas sus relaciones sociales".

El tema de los salarios ya fue abordado por los escolásticos del siglo XVI como Luis de Molina o Henrique de Villalobos como un tema de justicia conmutativa. La teoría del "salario justo" descansa en la voluntariedad, el libre consentimiento, excluyendo todo tipo de fraude o engaño. En este sentido hay que señalar, como ha apuntado Rafael Termes $^{33}$, que la necesidad del trabajador no determina el salario, así como la necesidad del propietario no determina el precio del alquiler o del arrendamiento. El salario justo es el que resulta de la libre negociación entre las dos partes. En cualquier caso dejar sujeto al trabajador a la autonomía privada y al mercado sería dejarlo sin protección. En las relaciones de trabajo se dan vínculos de poder donde el trabajador es la parte más débil y es necesario un marco jurídico que regule estas relaciones laborales, de ahí que el derecho laboral establezca unos mínimos irrenunciables en diversas materias y entre ellas la remuneración que protejan al trabajador en esa relación asimétrica puesto que lo contrario sería cosificar al trabajador y convertirle en un objeto de derecho disponible al mejor postor. En este sentido podemos observar como ejemplo, cómo la última reforma laboral del gobierno de España de 10 de febrero de 2012 ha servido para dar mayor poder al empresariado y a la vez ha traído consecuencias detestables para el trabajador como la simplificación y facilitación del despido, así como mayor precariedad, salarios bajos, inestabilidad y rotación de los trabajadores, de tal forma que, como señala Martínez Veiga ${ }^{34}$ la "flexibilidad" se ha convertido en la quintaesencia del espíritu capitalista del posfordismo donde se reniega de cualquier rigidez.

\footnotetext{
${ }^{31}$ GODELIER, M. (1967). Racionalidad e irracionalidad en la economía. México: Siglo XXI.

32 GODELIER, M. (1977). Teoría marxista de las sociedades precapitalistas. Barcelona: Laia.

33 TERMES, R. (2001). Antropología del capitalismo: un debate abierto. Madrid: Ediciones Rialp, p.123.

${ }^{34}$ VEIGA, U. M. (2016). "La reforma laboral de 2012 y el aumento del despido y desempleo en España". Revista Andaluza de Antropología, 11, pp. 44-66.
} 
En cuanto al salario, Marx ${ }^{35}$ decía que el salario es una consecuencia inmediata del trabajo alienado, y éste es la causa inmediata de la propiedad privada. En consecuencia, si se cae un lado, debe caer también el otro. En este sentido la interpretación que podemos hacer es que Marx quería decir que si desaparece la propiedad privada debe desaparecer lo que la genera, que no es otra cosa que el trabajo alienado, el trabajo asalariado. Por eso Marx también llama al trabajador asalariado esclavo, es esclavo de un dominio abstracto en donde es el mismo trabajador quien llama a la fábrica, entra en la propiedad privada y vende su fuerza de trabajo, y allí se encuentra inmerso en la producción capitalista donde no hay autodeterminación ni libertad, no importa qué utilidad presta a la humanidad porque todo se realiza por y para la ganancia capitalista $^{36}$. Como decía Harvey ${ }^{37}$, el dinero confiere el privilegio del poder sobre los otros: podemos comprar su tiempo de trabajo o sus servicios y hasta construir relaciones de dominación sistemáticas sobre las clases explotadas, simplemente, a través del poder del dinero.

En cualquier caso, actualmente en las sociedades desarrolladas el trabajo asalariado desempeña un papel central en la vida de las personas. Los ingresos que el trabajador obtiene son determinantes para su calidad de vida y como indica Watson ${ }^{38}$, su implicación en el trabajo es un elemento para la construcción de su identidad personal.

\section{LA ORGANIZACIÓN DEL TRABAJO: LA CULTURA DE LAS ORGANIZACIONES.}

En sociedades que practicaban la caza y la recolección o la horticultura apenas existe una organización formal al estilo occidental del trabajo, aunque ya vimos anteriormente cómo muestra Malinowski ${ }^{39}$ que en algunas sociedades de este tipo sí la hay, pero a medida que las sociedades son más complejas e industrializadas es necesario un mayor grado de organización del trabajo y por lo tanto una mayor especialización ocupacional.

Desde un punto de vista formal, una organización, según Schein ${ }^{40}$, que sitúa el término del griego organon, o sea, instrumento o herramienta, es la coordinación

\footnotetext{
${ }^{35}$ MARX, K. 2004 [1844]. Manuscritos económico-filosóficos de 1844. Buenos Aires, Colihue.

${ }^{36}$ CONTARTESE, D.L. y LOPEZ, N.A. (2012) “Alienación: la dignidad negada. En busca de caminos para cambiar el mundo". Revista Herramienta.

${ }^{37}$ HARVEY, D. (1998). La condición de la posmodernidad. Buenos Aires: Amorrortu, p.122.

38 WATSON, T. (2002): "Professions and professionalism. Should we jump off the bandwagon, better to study where it is going?". International Studies of Management and Organization,.32(2), pp. 93-15.

39 MALINOWSKI, B. (1986). Los Argonautas del Pacifico Occidental. Barcelona: Península, p.69.

40 SCHEIN, E. H. (1993). Psicología de la Organización. México: Prentice Hall. p.14.
} 
planificada de las actividades de un grupo de personas para procurar el logro de un objetivo explícito y común, a través de la división del trabajo y funciones, y a través de una jerarquía y responsabilidad. Es necesario en todo caso distinguir los diferentes agrupamientos que aparecen en una interacción de personas que desarrollan una cultura propia de cara a la consecución de unos objetivos. Como apunta Aguirre Baztán ${ }^{41}$, en todo agrupamiento se crea una membrana de identidad donde la interacción de sus miembros genera una cultura propia, una trama simbólica de significados compartidos que vertebra al grupo y le permite comunicarse. Siguiendo a este autor podemos dividir dichos agrupamientos en grupos, organizaciones e instituciones, con características particulares para cada uno de ellos y donde el grupo sería una entidad menor entendida como una formación subcultural que formaría parte de un ente superior llamado organización y englobado en la cultura de ésta. De la misma manera que uno o más grupos pueden transformarse en una organización, una organización puede convertirse en una institución donde existe una necesidad de permanencia que se afirma en su trayectoria desde el pasado. Por otra parte y como indica Kaës ${ }^{42}$, la institución es el conjunto de las formas y las estructuras sociales constituidas por la ley y la costumbre que regula nuestras relaciones y se inscribe en la permanencia. En contraposición a las organizaciones cerradas donde arquitectónica o psicológicamente su interior recela de su exterior, en las organizaciones abiertas, el interior depende del exterior y es ahí donde debería colocarse la empresa. De hecho el concepto de organización abierta surge en la Revolución Industrial del siglo XIX donde aparece realmente el término de división del trabajo como elemento fundamental de organización para conseguir los fines de forma eficaz. En cuanto al concepto de "cultura de la empresa", la acepción más aceptada es la que nos proporciona $\operatorname{Schein}^{43}$ que la define como un conjunto de elementos interactivos fundamentales, generados y compartidos por los miembros de una organización al tratar de conseguir la misión que da sentido a su existencia como empresa. La empresa es una cultura que se asienta en dos pilares: su "misión" o razón de ser y el liderazgo que la sustenta, el conductor de la cultura de la empresa. Por tanto, cambiar la cultura es como cambiar la empresa. En este punto es interesante rescatar los cinco elementos fundamentales que propone Aguirre Baztán ${ }^{44}$ como indicadores básicos portadores de su identidad cultural: etnohistoria, creencias, valores, comunicación y productos que deben estar cohesionados puesto que aunque

\footnotetext{
${ }^{41}$ AGUIRRE BAZTÁN, A. (2004). La cultura de las organizaciones. Barcelona: Ariel. p.29.

${ }^{42}$ KAËS, R. (1989). La institución y las instituciones: estudios psicoanalíticos. Buenos Aires: Paidós, p. 22.

43 SCHEIN, E.H. (1988) La cultura empresarial y el liderazgo, Barcelona: Plaza y Janés.

44 AGUIRRE BAZTÁN, Á. (2003). “¿Qué es la cultura de la empresa?” AIBR, 27, p. 3.
} 
haya la tentación de gestionar las partes de la cultura de la empresa no se puede olvidar que son un todo.

Como vemos, entonces, la cultura es un concepto clave que define la actividad e identidad de los grupos humanos y por tanto, de una empresa, de tal forma que no hay grupo sin cultura ni cultura sin grupo. Desde un punto de vista antropológico la noción de referencia hoy en día sigue siendo la de Taylor ${ }^{45}$ quien en su obra "Primitive culture" (1871) la define como "la totalidad compleja que incluye conocimientos, creencias, arte, ley, moral, costumbres y cualquier otra capacidad y hábitos adquiridos por el hombre y en cuanto a miembro de una sociedad". Mientras, frente a esta concepción evolucionista de Edward B. Taylor nos encontramos con el concepto particularista de Franz Boas nacido del estudio de los aislamientos geográfico-políticos, cercana a la idea del romanticismo alemán de kultur y de la idea de Sapir en el que el concepto de cultura se refiere más a lo espiritual de los grupos humanos sobre el que se basará posteriormente la antropología cultural norteamericana. Dentro del concepto de cultura que surge de la realidad urbano-industrial actual observamos una nueva realidad del pluralismo grupal y organizacional al que se adscriben los individuos. Siguiendo a Esteva ${ }^{46}$, podemos definir la cultura como "el modo de pensar organizado de los individuos de una sociedad, en orden a producir actividades sociales coherentes, tanto de acción material como de acción espiritual". Por otra parte para Buxó ${ }^{47}$ "la cultura es el sistema de conocimiento a partir de cuyos significados el ser humano tamiza y selecciona su comprensión de la realidad en sentido amplio, así como interpreta y regula los hechos y los datos del comportamiento social". Por lo tanto, el comportamiento humano de cada grupo encuentra sentido en el modelo de realidad que cada cultura produce.

El concepto de "cultura organizacional" es mucho más tardío, y se debe a Pettigrew (1979). A partir del concepto de cultura usada tradicionalmente por los antropólogos referida a las comunidades "primitivas" se ha querido formular también desde el mundo de la empresa un concepto que sirviera también de referencia para las realidades culturales urbanas y por lo tanto de las organizaciones que es donde se enmarcan. Así Aguirre ${ }^{48}$ define la cultura organizacional como "el conjunto de elementos interactivos fundamentales generados y compartidos por los miembros de la

\footnotetext{
45 TYLOR, E. B., Suárez, M., \& Radin, P. (1981). [1871] Cultura primitiva. Madrid: Ayuso.

46 ESTEVA FABREGAT, C. (1984). "El concepto de cultura”. En Fernández-Martorell, M. (Dir). Sobre el concepto de cultura. Barcelona: Editorial Mitre, p.65.

${ }^{47}$ BUXÓ, M. J. (1984). "La cultura en el ámbito de la cognición”. En Fernández-Martorell, M. (Dir). Sobre el concepto de cultura. Barcelona: Editorial Mitre, p.33.

48 AGUIRRE BAZTÁN, A. (2004). La cultura de las organizaciones. Barcelona: Ariel. p.159.
} 
organización al tratar de conseguir la misión que da sentido a su existencia”. Para Ribes ${ }^{49}$ entender la organización como persona grupal (empresa, institución, sociedad, grupo religioso...) nos permite configurar el modelo antropológico, pudiendo afirmar que las "personas grupales" también tienen memoria, entendimiento, voluntad, afectividad, motivaciones, ética, misión, es decir, cualidades análogas a las individuales y que no son partes sino aspectos de una realidad unitaria, de este modo, las personas grupales (organizaciones) tiene tres elementos relevantes: la misión, la cultura y el éxito.

En este punto es pertinente una rápida referencia a la literatura sobre las organizaciones, donde nos encontramos con tres clásicos de la organización: En primer lugar, F.W. Taylor y la "organización científica del trabajo", quien argumenta una doble consideración del hombre, el que piensa y dirige que estaría formado por personas inteligentes con capacidad de mandar y tomar decisiones y el que trabaja como una máquina formado por obreros y masas de trabajadores de baja cualificación y que estarían colocado en un estrato inferior. El taylorismo ha sido una ideología ampliamente criticada por los movimientos sociales reivindicativos puesto que ha sido y es una ideología básica del capitalismo salvaje que atenta contra la dignidad de las personas trabajadoras. De hecho para Taylor la baja rentabilidad de una empresa siempre era culpa de la "naturaleza holgazana" del trabajador. En este sentido Fernánde $z^{50}$ considera que parece más que discutible llamar organización científica a lo que no es más que un sistema para aumentar el rendimiento del utillaje y la mano de obra. Rendimiento y eficacia, que según Esteva Fabregat ${ }^{51}$ han sido los valores perseguidos por la ideología taylorista de la producción a través de la cronometrada división del trabajo humano y el mecánico, lo que ha producido una intensa devaluación de la identidad del yo como obrero, que ha derivado hacia fuertes frustraciones de su personalidad, a la monotonía y a la pérdida de la "alegría creadora del trabajo".

En segundo lugar, con Fayol ${ }^{52}$ y la "organización formal", se establece una estructura organizativa piramidal muy jerarquizada en la que el trabajo se divide en función del nivel que ocupa cada individuo en la escala sin cuestionar la autoridad única y jerárquica, dándose por lo tanto una verticalidad formal en la línea de Taylor pero aportando la departamentalización y la especialización. Fayol establece unos principios que se basan en la división del trabajo, la autoridad y responsabilidad, la disciplina, la unidad de mando y dirección. Y afirma que estos principios "apuntan

\footnotetext{
49 RIBES PONS, Á. (2000). "La antropología de la misión”. Harvard Deusto Business Review, 97, pp. 48-53.

${ }^{50}$ FERNÁNDEZ, A. R. (2014). Psicología de las organizaciones. Barcelona: Editorial UOC.

${ }^{51}$ ESTEVA FABREGAT, C. (1984). Antropología industrial. Barcelona: Anthropos Editorial, pp. $252-253$.

${ }^{52}$ FAYOL, H., \& TAYLOR, F. W. (1987). Administración industrial y general. Barcelona: Orbis.
} 
al éxito de asociaciones de individuos y a la satisfacción de intereses económicos". Esta corriente también ha sido ampliamente criticada desde el sindicalismo y el socialismo marxista. Por último, Max Weber ${ }^{53}$ y la escuela burocrática establecen que la burocracia es racionalidad y la forma más eficiente de organización. A través de un conjunto de reglas y la sumisión del personal a las mismas más que a la voluntad del jefe se realiza el ejercicio racional del poder. Afirma que la legitimación de imponer a los subordinados unas reglas puede ser triple: carismática, tradicional y legal. La organización burocrática es precisa, estructurada y actúa solo por los intereses de la organización. Las principales críticas a la organización burocrática se refieren a su mecanicidad, poca falta de adaptación a los cambios, a su falta de creatividad y flexibilidad que provoca en los trabajadores desmotivación y falta de participación. En todo caso, autores de hoy en día como Paul du Gay ${ }^{54}$ realizan una defensa de la burocracia como una institución fundamental para el mantenimiento de cualquier régimen democrático, alertando de los riesgos de prescindir de ella.

Desde un punto de vista antropológico tienen gran importancia en este tema los estudios de Elton Mayo y W.L. Warner de la Escuela de Chicago, que suponen el punto de partida en la "Escuela de las relaciones humanas". Mayo describe en "Los problemas sociales de la civilización industrial" (1945) sus hallazgos sobre la fatiga física laboral y la fatiga psíquica, constatando que la remuneración no es suficiente para motivar e incentivar al individuo, evidenciando que las variables sociales como la edad, sexo, estilo de liderazgo o participación son más decisivas en la producción que las variables básicas como la alimentación o los factores físicos como la iluminación, la humedad o la temperatura entre otros. Por otra parte, los estudios antropológicos de Warner ${ }^{55}$ pusieron de manifiesto que el clima social era determinante en la calidad y cantidad del rendimiento productivo, y la cultura organizacional y el clima laboral aparecen como dos nuevas realidades en el tratamiento de los grupos organizacionales. Estableció así mismo, la relación entre los elementos extraorganizacionales o factores socio-culturales externos como la raza, la religión o la familia y la cultura organizacional, todo ello de una gran importancia para comprender el comportamiento laboral. En este sentido fue fundamental la aplicación por parte de Warner, como ya había realizado en sus estudios sobre la organización del parentesco en una tribu aborigen, del método de campo a este tipo de organizaciones concibiendo la fábrica como si fuera una micro sociedad. Esto le permitió comprobar la validez e importancia

\footnotetext{
53 WEBER, M. (2015). ¿Qué es la burocracia?. Santa Fe, Asociación Civil Mírame Bien.

${ }^{54}$ DU GAY, P. (2014). "En elogio de la burocracia. Weber. Organización. Ética”. Reis, 146, pp. 239-254.

55 WARNER, W. L., \& LUNT, P. S. (1942). "The social life of a modern community". American Sociological Review, 7, pp. 263-271.
} 
del método aplicado a este nuevo campo y sirvió para introducir en el mundo de la empresa algunos conceptos fundamentales de la antropología con los que describir la realidad organizacional ${ }^{56}$. Es interesante observar, como apunta Jordi Roca ${ }^{57}$, que en la década de los 50 en Estados Unidos, existía la creencia generalizada entre los investigadores de que los que hacían trabajo de campo en su propio país en la línea de investigación del trabajo industrial (Gardner, Warner, Richardson, Whyte o Chapel) no eran auténticos antropólogos, en contraste con los que lo realizaban en el extranjero, lo que provocó que la antropología cediera terreno a otras disciplinas sociales como la sociología, la psicología, la economía, la administración de empresas o la ciencia política a pesar de que sus aportaciones a la investigación aplicada a la industria mediante el uso de la metodología etnográfica y cualitativa, combinando la entrevista y la observación y aportando el concepto de cultura al ámbito de las organizaciones habían sido transcendentales y muy significativas.

No hay que olvidar tampoco a los "teóricos de grupo" con sus clásicos estudios sobre el liderazgo. Llegaron a la conclusión de que aunque la productividad puede ser tan alta en organizaciones dirigidas con un liderazgo autoritario, el liderazgo democrático produce muchas menos tensiones y conflictos que en el primero, creándose un mejor clima laboral y mayor satisfacción en los trabajadores. En este grupo encontramos a Lewin, Lippitt y Katz entre otros. En el campo del estudio de los grupos pequeños hay que destacar a Charles Cooley ${ }^{58}$, quien distingue los grupos primarios, caracterizados por relaciones íntimas y afectivas cara a cara, de los secundarios, donde las relaciones son más anónimas e impersonales; y también a Kurt $\operatorname{Lewin}^{59}$ que examinó a estos grupos reducidos desde el punto de vista de su dinámica y la eficacia de la participación en la toma de decisiones. Para Lucas Marín ${ }^{60}$ las relaciones sociales informales que dan lugar a los grupos informales surgen en la empresa y tienden a crearse por una parte a través de la ampliación lógica de las relaciones formales y por otra por la búsqueda por parte de los individuos de situaciones de expansión afectiva, juegos o bromas con un cierto paralelismo con las de trabajo.

A partir de los años 70 con las investigaciones de antropólogos como Lauda Nader en Estados Unidos o Esteva-Fabregat y Ángel Palerm en México se produce una vuelta a los estudios antropológicos sobre el trabajo industrial, con los estudios

\footnotetext{
${ }_{56}$ TRUJILLO, J. T. (2010). “Antropología en México y España: industria, trabajo y organizaciones”. Virajes, p. 197.

${ }^{57}$ ROCA, J. (1999). "De la (im) pertinencia del obrero como objeto de estudio de la antropología social”. Política y Sociedad, 31, p. 201.

58 COOLEY, C. H. (1956). Social organization. New Jersey: Transaction Publishers.

59 LEWIN, K. (1951). Field theory in social science: Selected Theoretical Papers. New York: Harper \& Brothers.

${ }^{60}$ LUCAS MARÍN, A. (1981). Sociología de la empresa. Madrid: Ibérico Europea de Ediciones.
} 
de antropología del trabajo, de la cultura organizacional y del análisis de procesos organizacionales, "redescubriéndose" ese interés por parte de la antropología del mundo del trabajo y del estudio de las organizaciones industriales.

Mientras el interés por parte de la antropología por el mundo laboral industrial es una consecuencia en el tiempo de los procesos avanzados de industrialización de los diferentes países, en el caso de la antropología española hay una diferencia significativa: los antropólogos españoles parecen buscar más el estudio de los últimos primitivos más que las nuevas formas de organización o producción industrial, por lo que los estudios sobre el campesinado constituyen la mayoría de los estudios en este sentido, poniendo el interés más en procesos laborales o prácticas tradicionales, y en las sociedades preindustriales, rurales y campesinas y dejando el estudio de las sociedades "complejas", industriales y urbanas casi en manos exclusivamente de la sociología, convirtiéndose en cierto modo en aquellos años en un tabú el estudio de la antropología de los aspectos relacionados con la producción industrial. Posteriormente y a partir de los años 70 aparece este interés por la antropología industrial de la mano de C. Esteva, I. Moreno, y P. Palenzuela y J. Roca, aunque algunos de estos autores superando el término industrial y acuñando el concepto de "culturas del trabajo", y refiriéndose a la antropología del trabajo más en la línea de la etnología francesa y la sociología italiana. Como señala Isidoro Moreno ${ }^{61}$, para la sociología italiana la significación del trabajo se encuentra en el lugar que este ocupa en la vida de los individuos y su influencia en la vida social. Por cultura del trabajo entienden el conjunto de las representaciones e ideologías existentes en el trabajo. Por el contrario, los etnólogos franceses centraban su atención sobre la "cultura técnica", considerada como los conocimientos y saberes de los trabajadores en una actividad concreta; la división técnica y social del trabajo y sus formas de organización; la construcción de identidades locales y la relación entre la evolución tecnológica, las nuevas relaciones sociales y las nuevas prácticas culturales.

En cualquier caso, para Roca ${ }^{62}$ existe una escasa o deficiente relación entre antropología y empresa que se halla en la desconfianza mutua, que emana del desconocimiento entre ambos mundos; por una parte "un antropólogo metido en un escenario empresarial semeja a alguien embarrancado en zona pantanosa" y por otra parte, uno de los rasgos que definen en buena medida la antropología de la empresa es su carácter aplicado por lo que no es extraño que la antropología, que es una de las

${ }^{61}$ MORENO, I. (1997). "Trabajo. Ideologías sobre el trabajo y culturas del trabajo". Revista Andaluza de Relaciones Laborales, 3, pp. 9-28.

${ }^{62}$ ROCA, J. (2001). “¿Antropólogos en la empresa?: a propósito de la (mal) llamada cultura de empresa”. Etnográfica, 5, p. 75 . 
profesiones más academizadas que existen, no mire con entusiasmo a una subdisciplina eminentemente aplicada. En este sentido cabría preguntarse por el código ético o deontológico de la disciplina y qué posiciones debe recoger y hasta dónde debe llegar la ética personal del profesional.

En el campo de la antropología aplicada e investigación acción participativa no podemos dejar de mencionar a Davyd Greenwood ${ }^{63}$ y su trabajo sobre las culturas de la empresa cooperativa FAGOR del grupo Mondragón, que se promovió en un contexto de recesión económica y reconversión industrial en los años ochenta en España para analizar las realidades sociales de la empresa. En su estudio demostró que uno de los mayores defectos de la empresa era la gestión de los recursos humanos, realizado desde un punto de vista autoritario y nada democrático, donde se manifestaba una gran diferencia entre los miembros del ejecutivo y los trabajadores manuales. La empresa culpaba a los trabajadores de ser apáticos, cuando el problema realmente estaba en la misma gestión de los recursos humanos. Según Greenwood, partiendo de la diversidad y complejidad de las experiencias concretas de los trabajadores y directivos, la metodología participativa permite vincular el análisis de estas empresas con la diversidad cultural superando la idea de que la cultura corporativa es una realidad homogénea o uniforme ${ }^{64}$. Para Greenwood ${ }^{65}$, la observación participante dentro de la investigación-acción llega a tener un significado distinto, es una investigación social entre un investigador profesional y los "dueños del problema". Ambos conceptos se conceptualizan desde el punto de vista de una investigación colaborativa y recíproca y de una iniciativa a favor del cambio social, para lo que es necesario el compromiso de participación de los propios dueños locales del problema. Todos los participantes son a la vez observadores que conjugan sus observaciones para conseguir unos resultados con éxito.

No quedaría completo el panorama si no habláramos del debate ético de la Antropología de la empresa. Cualquier trabajo de campo está expuesto a sesgos que condicionan la obtención de la información. En palabras de Sergio López ${ }^{66}$, el incumplimiento de las buenas prácticas investigadoras es un fenómeno que se puede polarizar de manera extrema, y que no solo perjudica a los informantes, sino también

${ }^{63}$ GREENWOOD, D. J., \& SANTOS, J. L. G. (Eds.). (1992). Industrial democracy as process: Participatory action research in the Fagor Cooperative Group of Mondragon. Assen: Van Gorcum.

${ }^{64}$ SÁNCHEZ MOLINA, R. (2009). La etnografia y sus aplicaciones: lecturas desde la antropología social y cultural. Madrid: Editorial Universitaria Ramón Areces. p.39.

${ }^{65}$ GREENWOOD, D. (2000). "De la observación a la investigación-acción partipativa: una visión crítica de las prácticas antropológicas”. Revista de antropología social, 9, pp. 34-35.

${ }^{66}$ LÓPEZ, S. (2017): Antropología de la empresa. Barcelona: Ediciones Bellaterra, pp. 229-233. 
a los investigadores y a las organizaciones. Para ello hay dos factores clave: por una parte no dañar a los informantes y obtener de ellos el consentimiento y respetar el anonimato puesto que muchas veces los informantes no son conscientes de los datos que nos están dando y del riesgo que para ellos puede tener que los transmitamos a sus superiores. Por otra parte está el "efecto del confesor", es decir, cuando la información que se ha descubierto es comprometedora o incluso delictiva y puede implicar por lo tanto riesgos para el informante y para el propio investigador. Por lo tanto, evaluar las cuestiones éticas del trabajo de los antropólogos implica considerar ambos riesgos.

Por último, hay que indicar que la economía global basada en las nuevas tecnologías de la información y la comunicación, va más allá de los trabajadores en masa. En palabras de Jeremy Rifkin ${ }^{67}$ mientras las élites sean necesarias para hacer funcionar la economía formal del futuro, cada vez menos trabajadores serán necesarios para fomentar la producción de bienes y servicios. La completa sustitución de los humanos por las máquinas deja a los trabajadores sin autodefinición válida o función social. Mientras tanto el papel de los gobiernos parece que se desvanece a manos de las empresas transnacionales ¿Estamos por lo tanto ante un nuevo contrato social?

\section{CONCLUSIONES.}

En esta investigación se ha señalado que el trabajo es la condición de la misma supervivencia del hombre, es la posibilidad de adecuar especialmente el entorno a sus necesidades. Desde la antigüedad el hombre ha realizado diferentes tareas para la supervivencia, empezando por la recolección de alimentos y, precisamente con el reparto de tareas nació la división del trabajo, primeramente una división sexual y posteriormente la división jerarquizada en función de las divisiones sociales. Posteriormente a esta división sexual del trabajo, la interacción entre el patriarcado y el capitalismo otorgó un valor diferencial al trabajo en función del género y que todavía es palmaria en nuestros días.

A lo largo de la Historia la relación entre trabajo y sistemas de producción ha sido diversa, pero siempre el hombre ha estado sujeto al trabajo de diferentes formas, desde la esclavitud, el vasallaje o el obrero proletario, siempre sujeto al dueño de los medios de producción. Pensadores como Marx, Engels, Durkheim o Weber son fundamentales para entender estas nuevas sociedades industriales de finales del XIX

\footnotetext{
${ }^{67}$ RIFKIN, J. (1996). El fin del trabajo. Nuevas tecnologías contra puestos de trabajo: el nacimiento de una nueva era. Barcelona: Paidos, p. 277.
} 
y principios del XX y las relaciones asimétricas entre obreros y patronos, donde la generación de riqueza iba acompañada de enormes desigualdades económicas, sociales y políticas que acrecentaron el conflicto entre las clases. A través de ese trabajo alienado el trabajador genera la relación con un trabajo que es ajeno a él. Posteriormente a través de la "organización científica del trabajo" (Tylor, Fayol, Stajanov, Ford) la deshumanización del trabajo fue casi total en aras de la productividad. Unos métodos de trabajo que como manifestamos a través de este estudio se repiten en pleno siglo XXI, con empleados altamente productivos sojuzgados por métodos de control y coerción simbólica.

Con todo ello surge el debate del salario considerado justo, donde no podemos dejar en manos de las partes (trabajador y empresario) la negociación de todas las condiciones laborales puesto que la parte más débil que es el trabajador, estaría en desventaja, por lo que se necesita un marco jurídico que regule esas relaciones y ponga los límites. A pesar de ello, hoy en día la flexibilidad laboral se ha convertido en la quintaesencia del espíritu capitalista y los empresarios abogan por una mayor desregulación que sin duda les beneficie. Como decía Godelier $(1967,1990)$ el modo de producción tiene un proceso doble, el trabajo como relación entre hombres y naturaleza y la relación de los hombres entre sí, y estas relaciones pueden ser más o menos solidarias o más o menos conflictivas.

Se ha tratado de la cultura de las organizaciones, como un agrupamiento donde se crea una membrana de identidad donde la interacción de sus miembros genera una cultura propia, una trama simbólica de significados compartidos que vertebra al grupo. La empresa es una cultura donde se asientan dos pilares, su misión y el liderazgo que la sustenta, porque no hay grupo sin cultura ni cultura sin grupo.

Desde nuestra consideración antropología se ha incidido en Mayo y Warner de la "Escuela de relaciones humanas" que aplicaron el método etnográfico en el estudio de las relaciones sociales en la empresa con resultados muy significativos, determinando que la cultura organizacional y el clima laboral o los factores socioculturales son de gran importancia para comprender el comportamiento laboral. En España, de la mano de antropólogos como Esteva, Moreno, Palenzuela o Roca, se han ido estudiando las "culturas del trabajo" en el propio contexto español en, como dice Roca, una relación entre laAntropología y la empresa de desconfianza mutua. En este sentido, la observación participante y la investigación-acción son fundamentales en la investigación social como apunta Greenwood para conseguir resultados con éxito. Por lo tanto podemos concebir la Antropología del Trabajo como una disciplina apasionante para investigar el mundo laboral, con retos desafiantes como el que apunta Rifkin en su obra "El fin 
del trabajo": no sabemos a dónde nos llevará la sustitución de los humanos por las máquinas que dejaría a los trabajadores sin función social.

\section{REFERENCIAS BIBLIOGRÁFICAS}

ABASOLO, O. \& MONTERO, J. (2012). “Trabajos: empleo, cuidado y división sexual del trabajo". Guía de ciudadanía con perspectiva de género. Madrid: Fuhem Ecosocial.

AGUIRRE BAZTÁN, Á. (2003). “Qué es la cultura de la empresa?” AIBR, 27, p.3.

AGUIRRE BAZTÁN, A. (2004). La cultura de las organizaciones. Barcelona: Ariel.

BOHANNAN, P. (1996). Para raros, nosotros. Madrid: Ediciones AKAL.

BOURDIEU, P. (1998). La dominatión masculine. Paris: Le Seuil.

BUXÓ, M. J. (1984). "La cultura en el ámbito de la cognición”. En Fernández-Martorell, M. (Dir). Sobre el concepto de cultura. Barcelona: Editorial Mitre, p.33.

CHAPLIN, C., CHAPLIN, C. (1936). Modern Times. Estados Unidos: United Artists.

COMAS D’ARGEMIR, D. (1995). Trabajo, género, cultura: la construcción de desigualdades entre hombres y mujeres. Barcelona: Icaria.

CONTARTESE, D.L. y LOPEZ, N.A. (2012) “Alienación: la dignidad negada. En busca de caminos para cambiar el mundo". Revista Herramienta.

COOLEY, C. H. (1956). Social organization. New Jersey: Transaction Publishers.

CUESTA ÁVILA, R. (2016). "Debates teóricos sobre el 'fin de la sociedad industrial' en el horizonte laboral del nuevo milenio". Revista Andaluza de Antropología, 11, pp. 215-248.

DU GAY, P. (2014). "En elogio de la burocracia. Weber. Organización. Ética”. Reis, 146, pp. 239-254.

DURKHEIM, E. (1987). La división del trabajo social (Vol. 39). Madrid: Ediciones Akal.

ENGELS, F. (2011). El papel del trabajo en la transformación del mono en hombre. Valencia: NoBooks Editorial.

ESTEVA FABREGAT, C. (1984). "El concepto de cultura”. En Fernández-Martorell, M. (Dir). Sobre el concepto de cultura. Barcelona: Editorial Mitre, p.65.

ESTEVA FABREGAT, C. (1984). Antropología industrial. Barcelona: Anthropos Editorial.

FAYOL, H., \& TAYLOR, F. W. (1987). Administración industrial y general. Barcelona: Orbis. FERNÁNDEZ, A. R. (2014). Psicología de las organizaciones. Barcelona: Editorial UOC.

GARCÍA GUAL, C. (1990). "En busca de los orígenes: algunas etimologías de términos económicos". Revista de economía, 5, p. 114.

GODELIER, M. (1967). Racionalidad e irracionalidad en la economía. México: Siglo XXI.

GODELIER, M. (1976). Antropología y economía. Barcelona: Anagrama.

GODELIER, M. (1977). Teoría marxista de las sociedades precapitalistas. Barcelona: Laia. 
GREENWOOD, D. (2000). De la observación a la investigación-acción partipativa: una visión crítica de las prácticas antropológicas. Revista de antropología social, 9, pp. 34-35.

GREENWOOD, D. J., \& SANTOS, J. L. G. (Eds.). (1992). Industrial democracy as process: Participatory action research in the Fagor Cooperative Group of Mondragon. Assen: Van Gorcum.

HARVEY, D. (1998). La condición de la posmodernidad. Buenos Aires: Amorrortu.

KAËS, R. (1989). La institución y las instituciones: estudios psicoanalíticos. Buenos Aires: Paidós.

LEWIN, K. (1951). Field theory in social science: Selected Theoretical Papers. New York: Harper \& Brothers.

LÓPEZ, S. (2017): Antropología de la empresa. Barcelona: Ediciones Bellaterra.

LOZANO, M.J. \& PALENZUELA. P. (2016). Trabajo y culturas del trabajo en la globalidad hegemónica. Revista Andaluza de Antropología, 11, pp. 1-15.

LUCAS MARÍN, A. (1981). Sociología de la empresa. Madrid: Ibérico Europea de Ediciones.

MALINOWSKI, B. (1986). Los Argonautas del Pacífico Occidental. Barcelona: Península.

MAREIRA ESTRADA, F. (2002). Trabajo Infantil: algunas consideraciones desde la antropología. Rev. austral cienc. soc, 6, pp. 113-124.

MARX, K. 2004 [1844]. Manuscritos económico-filosóficos de 1844. Buenos Aires, Colihue.

MOLINA, J. L. (2004). Manual de antropología económica. Bogotá: UAB.

MOORE, H. L. (2009). Antropología y feminismo. Madrid: Cátedra.

MORENO, I. (1997). “Trabajo. Ideologías sobre el trabajo y culturas del trabajo". Revista Andaluza de Relaciones Laborales, 3, pp. 9-28.

MUNÁRRIZ, L. Á. (2009). "La identidad personal en la Región de Murcia". Revista murciana de antropología, 16, pp. 309-324.

NAROTZKY, S. (2004). Antropología económica. Nuevas tendencias. Santa Cruz de Tenerife: Melusina.

OSBORNE, R. (1993). La construcción sexual de la realidad: un debate en la sociología contemporánea de la mujer. Valencia: Universitat de Valencia.

PARKER, M. y SLAUGHTER, J. (1988) Choosing Sides: Unions and the Team Concept, Detroit: Labor Notes.

RESKIN, B. F., \& PADAVIC, I. (1994). Men and women at work. Thousand Oaks: Pine Forge Press.

RIBES PONS, Á. (2000). “La antropología de la misión”. Harvard Deusto Business Review, 97, pp. 48-53.

RIEZNIK, P. (2001). “Trabajo, una definición antropológica”. Razón y revolución, 7, 1-21.

RIFKIN, J. (1996). El fin del trabajo. Nuevas tecnologías contra puestos de trabajo: el nacimiento de una nueva era. Barcelona: Paidos. 
ROCA, J. (1999). "De la (im) pertinencia del obrero como objeto de estudio de la antropología social”. Política y Sociedad, 31, 201.

ROCA, J. (2001). “¿Antropólogos en la empresa?: a propósito de la (mal) llamada cultura de empresa". Etnográfica, 5, p.75.

ROSALDO, M. Z. (1980). "The use and abuse of anthropology: reflections on feminism and cross-cultural understanding". Signs, 5(3), pp. 389-417.

SÁNCHEZ MOLINA, R. (2009). La etnografía y sus aplicaciones: lecturas desde la antropología social y cultural. Madrid: Editorial Universitaria Ramon Areces. p. 39.

SCHEIN, E. H. (1993). Psicología de la Organización. México: Prentice Hall. p. 14.

SCHEIN, E.H. (1988) La cultura empresarial y el liderazgo, Barcelona: Plaza y Janés.

SMITH, A. (1994). Investigación sobre la naturaleza y causas de la riqueza de las naciones. México: Fondo de Cultura Económica.

TYLOR, E. B., Suárez, M., \& Radin, P. (1981). [1871] Cultura primitiva. Madrid: Ayuso.

TAYLOR, F. W. (1969). Principios de la Administración Cientifica. México: Herrero Hnos.

TERMES, R. (2001). Antropología del capitalismo: un debate abierto. Madrid: Ediciones Rialp.

TRUJILLO, J. T. (2010). “Antropología en México y España: industria, trabajo y organizaciones”. Virajes, p. 197.

ULRICH, D. (1997). Recursos humanos champions. L'Hospitalet de Llobregat: Ediciones Granica SA.

VEIGA, U. M. (2016). "La reforma laboral de 2012 y el aumento del despido y desempleo en España". Revista Andaluza de Antropología, 11, pp. 44-66.

WARNER, W. L., \& LUNT, P. S. (1942). "The social life of a modern community". American Sociological Review, 7, pp. 263-271.

WATSON, T. (2002): "Professions and professionalism. Should we jump off the bandwagon, better to study where it is going?". International Studies of Management and Organization, 32, pp. 93-15.

WEBER, M. (2015). ¿Qué es la burocracia?. Santa Fe, Asociación Civil Mírame Bien.

WOLF, E. R. (1987). Europa y la gente sin historia. Madrid: Fondo de Cultura Económica. 